\title{
Therapy Switches in Fingolimod-Treated Patients with Multiple Sclerosis: Long-Term Experience from the German MS Registry
}

\author{
Niklas Frahm (1) - Firas Fneish (1) - David Ellenberger (1) - Peter Flachenecker - Friedemann Paul (D) - \\ Clemens Warnke (1) - Christoph Kleinschnitz · Tina Parciak • Dagmar Krefting · Kerstin Hellwig • \\ Judith Haas · Paulus S. Rommer (1) · Alexander Stahmann (1) · Uwe K. Zettl
}

Received: November 3, 2021 / Accepted: December 21, 2021 / Published online: January 12, 2022

(C) The Author(s) 2022

\begin{abstract}
Introductions: Therapy switches in patients with multiple sclerosis (MS) receiving treatment with fingolimod occur frequently in clinical practice but are not well represented in realworld data. The aim of this study was to identify and characterize treatment switches and reveal
\end{abstract}

Supplementary Information The online version contains supplementary material available at https:// doi.org/10.1007/s40120-021-00320-w.

N. Frahm ( $₫) \cdot$ F. Fneish · D. Ellenberger .

A. Stahmann

MS Forschungs- Und Projektentwicklungs-gGmbH

(MS Research and Project Development gGmbH

[MSFP]), Krausenstr. 50, 30171 Hannover, Germany

e-mail: frahm@msregister.de;

niklas.frahm@med.uni-rostock.de

F. Fneish

e-mail: fneish@msregister.de

D. Ellenberger

e-mail: ellenberger@msregister.de

A. Stahmann

e-mail: stahmann@msregister.de

N. Frahm · P. S. Rommer · U. K. Zettl

Neuroimmunological Section, Department of

Neurology, University Medical Center of Rostock,

Gehlsheimer Str. 20, 18147 Rostock, Germany

U. K. Zettl

e-mail: uwe.zettl@med.uni-rostock.de sociodemographic/clinical changes over time in fingolimod-treated people with MS (PwMS).

Methods: Data on 2536 fingolimod-treated PwMS extracted from the German MS Registry during different time periods were analyzed (2010-2019).

Results: Overall, 28.3\% of PwMS were treatment-naïve before fingolimod initiation. Interferon beta $(30.7 \%)$ was the most common prefingolimod treatment. Ocrelizumab (19.8\%) was the most frequent subsequent treatment in the 944 patients on fingolimod who switched. Between 2010 and 2019, median disease

\section{P. Flachenecker}

Neurological Rehabilitation Center Quellenhof, Kuranlagenallee 2, 75323 Bad Wildbad, Germany e-mail: peter.flachenecker@sana.de

\section{F. Paul}

Experimental and Clinical Research Center, Max Delbrueck Center for Molecular Medicine and Charité-Universitätsmedizin Berlin, Lindenberger Weg 80, 13125 Berlin, Germany

e-mail: Friedemann.Paul@charite.de

\section{Warnke}

Department of Neurology, Medical Faculty,

University Hospital of Cologne, Kerpener Str. 62, 50937 Cologne, Germany

e-mail: clemens.warnke@uk-koeln.de

C. Kleinschnitz

Department of Neurology and Center of

Translational and Behavioral Neurosciences (C-

TNBS), University Hospital Essen, Hufelandstr. 55, 45147 Essen, Germany

e-mail: Christoph.Kleinschnitz@uk-essen.de 
duration at fingolimod initiation decreased from 8.5 to 7.1 years $(p<0.001)$, and patients taking fingolimod for $\geq 1$ year after treatment initiation decreased from 89.6 to $80.5 \%$ $(p<0.001)$. Females $(p<0.001)$ and young patients $(p=0.003)$ showed a shorter time on fingolimod. The most frequent reason for switching was disease activity (relapse/MRI) despite treatment. The annualized relapse rate increased from 0.37 in patients on fingolimod to 0.47 after treatment cessation, decreasing to 0.19 after treatment with a subsequent diseasemodifying drug (DMD) was initiated.

Conclusion: Treatment switches from fingolimod to subsequent DMDs currently occur after shorter treatment durations than 10 years ago, possibly due to the growing treatment spectrum. Planning adequate washout periods is essential and should be done on an individualized basis.

Keywords: Multiple sclerosis; Fingolimod; Treatment switches; Rebound; Diseasemodifying drug

T. Parciak · D. Krefting

Department of Medical Informatics, University Medical Center Göttingen, Von-Siebold-Str. 3, 37075 Göttingen, Germany

T. Parciak

e-mail: tina.parciak@med.uni-goettingen.de

D. Krefting

e-mail: dagmar.krefting@med.uni-goettingen.de

K. Hellwig

Department of Neurology, St. Joseph and St.

Elisabeth Hospital-Ruhr University, Gudrunstr. 56,

44791 Bochum, Germany

e-mail: k.hellwig@klinikum-bochum.de

J. Haas

Deutsche Multiple Sklerose Gesellschaft,

Bundesverband e.V. (German Multiple Sclerosis

Society [DMSG], Federal Association), Krausenstr.

50, 30171 Hannover, Germany

e-mail: haas-heide@gmx.de

P. S. Rommer

Department of Neurology, Medical University of Vienna, Währinger Gürtel 18-20, 1090 Vienna, Austria

\section{Key Summary Points}

\section{Why carry out this study?}

Switching disease-modifying drugs (DMDs) plays an important role in the therapeutic management of multiple sclerosis (MS).

Analyzing the role of fingolimod as part of the MS treatment strategy is of utmost importance due to its high efficacy, potential side effects, and the occurrence of rebound events after cessation of treatment.

The aim of this study was to identify and characterize treatment switches, the reasons for these switches, and the predictors of drug switching in fingolimod-treated patients with MS based on real-world data over the period 2010-2019.

\section{What was learned from the study?}

Most patients were treated with interferon beta prior to being switched to fingolimod $(30.7 \%)$ or were treatment-naïve $(28.3 \%)$, whereas the monoclonal antibodies ocrelizumab (19.8\%) and natalizumab (19.1\%) were the most common follow-up therapies for patients who switched from fingolimod.

The duration of fingolimod treatment is currently shorter than stated in the postmarketing approval statement, and the range of alternative treatments is growing; therefore, appropriate washout periods need to be determined on an individual basis.

\section{INTRODUCTION}

Multiple sclerosis (MS) is the most common immune-mediated chronic neurological disease in young adults [1]. Due to demyelination 
processes, damage to oligodendrocytes, synapse loss, and active gliosis in the central nervous system, these patients may suffer from a wide variety of symptoms, including paresis, spasticity, coordination disorders, gastrointestinal and bladder dysfunction, pain, cognitive and emotional disorders, and fatigue [2, 3].

Disease-modifying drugs (DMDs) are the mainstay of treatment for people with MS (PwMS) to prevent relapses, disability progression, and subclinical disease activity [2]. According to data from the German MS Registry (GMSR), PwMS start DMD treatment at a median of 4 months after MS diagnosis [4]. DMDs range from moderately to highly effective drugs, depending on disease activity [5].

The DMD fingolimod, a sphingosine-1phosphate receptor (S1PR) modulator, inhibits lymphocyte egress from lymph nodes $[5,6]$. Fingolimod was approved by the US Food and Drug Administration (FDA) in 2010 and by the European Medicines Agency (EMA) in 2011 for the treatment of patients with relapsing MS forms, being the first oral DMD approved for the treatment of highly active relapsing-remitting MS (RRMS) [7, 8]. In 2018, fingolimod was also approved for use in children aged $\geq 10$ years. This drug is characterized by increased therapeutic efficacy, reduced relapse activity, and delayed disability progression compared to traditional injectable DMDs, such as interferon beta and glatiramer acetate $[9,10]$. However, it may cause severe adverse effects, sucha as increased infection risk, lymphopenia, leukopenia, atrioventricular block, and progressive multifocal leukoencephalopathy (PML) $[1,11]$.

Assessing the comparative efficacy and safety of DMDs is challenging in clinical practice as head-to-head studies are rare. Additionally, the results of pivotal trials cannot be readily extrapolated to real-world patient cohorts because the specific inclusion criteria are not met by the majority of PwMS being treated in real-world settings [12]. Treatment switches are common in the rapidly developing therapeutic landscape. Therefore, an analysis of treatment strategies and their potential impact on treatment outcomes is of great importance to clinicians. Fingolimod, as an integral part of the MS treatment spectrum, represents an interesting and important target for investigation due to its high clinical efficacy, potential severe adverse effects, and rebound issues. Therefore the aim of this study was to identify and characterize treatment switches, reasons for the treatment switches, and predictors of switching in fingolimod-treated PwMS based on real-world data outside of pivotal trials. Moreover, we focused on the sociodemographic and clinical characteristics of fingolimod users over time (2010-2019).

\section{METHODS}

\section{Data Acquisition}

In 2001, the German MS Society initiated the German MS Register (GMSR). The GMSR was registered with the German Register of Clinical Studies (Deutsches Register Klinischer Studien [DRKS]; No. DRKS00011257), and initial ethical approval was obtained by the institutional review board at the University of Würzburg (Permit No. 142/12). The aim was to collect comprehensive and comparable clinical, sociodemographic, and therapeutic data on PwMS in Germany and to support MS research [13]. In 2014, the GMSR underwent technical revisions to include more comprehensive data on DMDs, including treatment duration, DMD type, and reasons for DMD discontinuation/ switch [13]. The structure, data basis, and methods of data collection and management of the GMSR are described comprehensively in a paper by Ohle et al. [13]. Since 2014 and up to 17 October 2021, data on 35,932 PwMS have been entered into the database. Of these PsMS, 2536 had received at least one fingolimod treatment between 2010 and 2019, with a minimum follow-up of 6 months, had a diagnosis of RRMS, and had a complete baseline demographic dataset available for assessment (see Fig. 1).

In this retrospective cohort study, PwMS were analyzed for sociodemographic, clinical, and treatment characteristics from 2010 to 2019. We divided the PwMS into three subgroups according to the time of their 


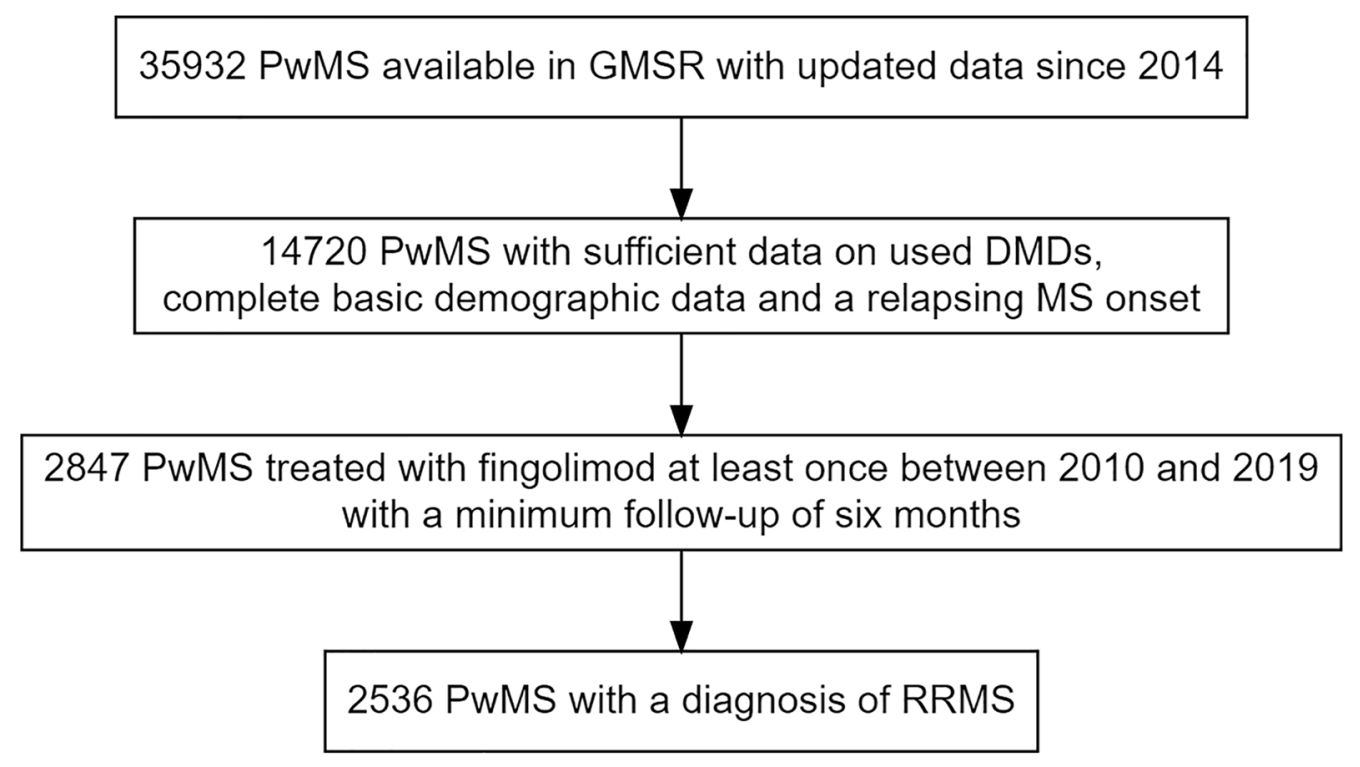

Fig. 1 Flow chart of patient selection for inclusion in the analysis. At the date of patient selection, the GMSR contained data on 35,932 PwMS. For the analysis, we selected PwMS with relapsing onset, a complete documentation of demographic data as well as DMD use, and at least one fingolimod treatment between 2010 and 2019, with a minimum follow-up of 6 months. Of these 2847

fingolimod treatment initiation. The first period was set from 2010 to 2013 and covers the approval of fingolimod in the European Union in 2011 including compassionate use in Germany in 2010 as well as the post-approval period during which time the high-efficacy DMD alemtuzumab (2013) and the modest-/moderate-efficacy DMD teriflunomide (2013) were also approved [7, 14-16]. The second period was set from 2014 to 2016 due to the approval of further DMDs for RRMS treatment during this time period, i.e., dimethyl fumarate in 2014 and daclizumab in 2016 [17, 18]. The third period covered 2017 to 2019, during which time the new high-efficacy DMDs cladribine (2017) and ocrelizumab (2018) were approved to treat RRMS $[16,19,20]$. Another factor considered in determining the three periods was the comparability regarding the sizes of the patient groups and the length of the time periods.
PwMS, data analysis was performed in patients who had a diagnosis of RRMS at the initiation of fingolimod treatment $(N=2536)$. DMD Disease-modifying drug, GMSR German MS Registry, MS multiple sclerosis, $N$ number of patients, PwMS people with MS, RRMS relapsing-remitting MS,

\section{Statistical Analysis}

Disease duration was defined as time between the symptom onset of MS and initiation of treatment with fingolimod. Washout periods were defined as the time between the end date of fingolimod treatment and the start date of the subsequent DMD. We only included PwMS with complete information (at least month and year) on the start and the end of fingolimod treatment in the washout analysis.

Alluvial graphs were used to visualize the frequencies of DMDs before and after fingolimod treatment. Chi-square and Kruskal-Wallis tests were performed to compare patient cohorts in the respective periods. Annualized relapse rates (ARRs: number of clinically defined relapses per observation period in years) of PwMS were calculated for the duration of treatment with fingolimod, washout periods, and the subsequent DMD. Cox regression was used to examine the association of sex, age, degree of disability, and length of 
Table 1 Clinical and demographic data of the patients included in the study

\begin{tabular}{|c|c|c|}
\hline Clinical and demographic characteristics & Patient values & \\
\hline Total number of patients, $N$ & 2536 & \\
\hline Female, $N(\%)^{a}$ & $1818(71.7)$ & \\
\hline Male, $N(\%)^{\mathrm{a}}$ & $718(28.3)$ & \\
\hline Age at MS symptom onset (years) & $29.6 \pm 9.4^{\mathrm{d}}$ & $5.6-66.8^{\mathrm{e}}$ \\
\hline Age at start of fingolimod (years) & $39.1 \pm 10.5^{\mathrm{d}}$ & $15.2-73.9^{\mathrm{e}}$ \\
\hline Partnership status & $\begin{array}{l}\text { At start of fingolimod } \\
\qquad(N=1767)^{c}\end{array}$ & $\begin{array}{l}\text { At end of fingolimod } \\
\qquad(N=1793)^{\mathrm{a}}\end{array}$ \\
\hline Single, $N(\%)$ & $523(29.6)$ & $503(28.1)$ \\
\hline Any partnership, $N(\%)$ & $1244(70.4)$ & $1290(71.9)$ \\
\hline Employment status & $\begin{array}{l}\text { At start of fingolimod } \\
\qquad(N=1708)^{c}\end{array}$ & $\begin{array}{l}\text { At end of fingolimod }(N= \\
1740)^{\mathrm{a}}\end{array}$ \\
\hline In training, $N(\%)$ & $86(5.0)$ & $57(3.3)$ \\
\hline \multirow[t]{3}{*}{ Employed, $N(\%)$} & 1092 (63.9): & $1085(62.4):$ \\
\hline & - Full time: 770 (45.1) & - Full time: 759 (43.6) \\
\hline & - Part time: 322 (18.9) & - Part time: 326 (18.7) \\
\hline \multirow[t]{3}{*}{ Retired, $N(\%)$} & 369 (21.6): & 451 (25.9): \\
\hline & - Disability: 315 (18.4) & - Disability: 386 (22.2) \\
\hline & - Old age: 54 (3.2) & - Old age: $65(3.7)$ \\
\hline Other, $N(\%)$ & $161(9.4)$ & $147(8.4)$ \\
\hline Educational level & $\begin{array}{l}\text { At start of fingolimod } \\
\qquad(N=1355)^{c}\end{array}$ & $\begin{array}{l}\text { At end of fingolimod } \\
\qquad(N=1580)^{\mathrm{a}}\end{array}$ \\
\hline NSCE, $N(\%)$ & $13(1.0)$ & $13(0.8)$ \\
\hline In training, $N(\%)$ & $2(0.1)$ & $2(0.1)$ \\
\hline CSE/GCSE, $N(\%)$ & $858(63.3)$ & $991(62.7)$ \\
\hline Advanced technical college entrance qualification, $N(\%)$ & $113(8.3)$ & $141(8.9)$ \\
\hline A level, $N(\%)$ & $369(27.2)$ & $433(27.4)$ \\
\hline Other variables & $\begin{array}{l}\text { Median (25\% quartile, } 75 \% \\
\text { quartile) }\end{array}$ & Range \\
\hline Disease duration from MS onset ${ }^{\mathrm{b}}$ (years) $(N=2407)$ & $7.6(3.4,14.0)$ & $0.0-43.8$ \\
\hline Disease duration from MS diagnosis ${ }^{\mathrm{b}}$ (years) $(N=2463)$ & $5.8(2.4,11.5)$ & $0.0-41.8$ \\
\hline Duration of fingolimod treatment (years) $(N=2536)$ & $3.2(1.5,5.8)$ & $0.0-11.1$ \\
\hline EDSS at start of fingolimod $(N=1158)^{c}$ & $2.0(1.5,3.5)$ & $0.0-8.0$ \\
\hline
\end{tabular}


Table 1 continued

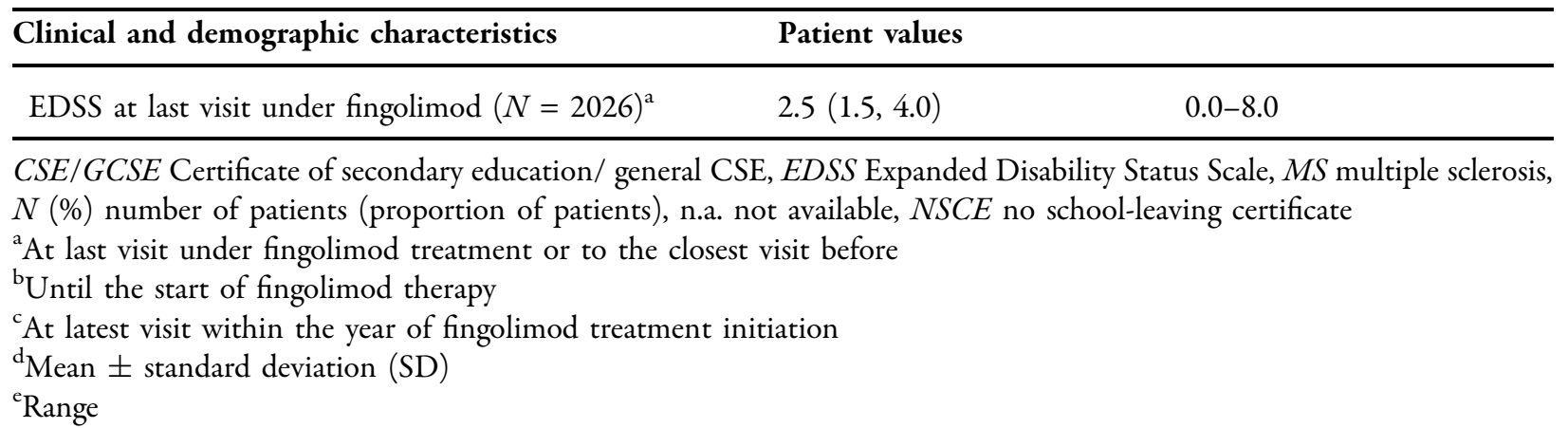

the washout period with (1) treatment duration of fingolimod and (2) the time to the first MS relapse during the washout period, as well as during treatment with the subsequent DMD. Data analysis and transformation were performed, and figures were created using the $\mathrm{R}$ v4.0 software program (R Foundation for Statistical Computing, Vienna, Austria).

\section{RESULTS}

\section{Study Population Over Time}

The clinical and sociodemographic characteristics of the patients included in the analysis are shown in Table 1. A detailed overview of available patient data is described in Electronic Supplementary Material (ESM) Fig. S1.

The results of our analysis of the sociodemographic, clinical, and therapeutic characteristics of fingolimod patients for the three periods (2010-2013, 2014-2016, and 2017-2019) are shown in Table 2 . The disability level at the start of fingolimod treatment decreased over time from a median Expanded Disability Status Scale (EDSS) score of 2.5 (2010-2013) to $2.0(2017-2019) \quad(p<0.001)$. The proportion of patients treated with fingolimod for $\geq 1$ year decreased by $9 \%$ from the first to the third period $(p<0.001)$. The proportion of treatment-naïve PwMS starting fingolimod was $23.0 \%$ in the first period (2010-2013), peaked at $33.3 \%$ during the 2014-2016 period, and decreased again to $28.0 \%$ during the most recent period $(p<0.001)$. The median disease duration at initiation of fingolimod treatment decreased from 8.5 to 7.1 years $(p<0.001)$. There were no statistically significant differences for sex ratios and age at fingolimod initiation over time ( $p \geq$ 0.282 ).

\section{DMDs Used Prior to Fingolimod Initiation}

Of the 2536 patients with RRMS who were treated with fingolimod, $28.3 \%$ were treatmentnaïve prior to fingolimod initiation. During the 2010-2019 period, most patients switched from interferon beta to fingolimod $(N=778,30.7 \%)$ (Fig. 2).

\section{Subsequent DMD Utilization After Fingolimod}

Around $58 \% \quad(N=1469)$ of the 2536 PwMS continued their fingolimod therapy until the end of the observation period (median followup 4.5 years, range $0.5-11.1$ years), while $37.2 \%$ $(N=944)$ switched to another DMD, and $4.9 \%$ $(N=123)$ stopped DMD treatment and did not re-start therapy until the end of the observation period (median 9.8 months, range 0.02-103.7 months), see Fig. 2. The distribution of subsequent DMDs over time is summarized in ESM Table S1. The most frequent follow-up treatments of the 944 PwMS who switched treatment were ocrelizumab (19.8\%), natalizumab (19.1\%), or dimethyl fumarate (11.2\%). Of those patients who switched, 85 paused their use of fingolimod during the observation period and but resumed the treatment after a median 
interruption of 4.2 (range 0.7-33.2) months. Combined drug utilization before and after fingolimod is shown in ESM Document S1. Within the following 2 years of subsequent DMD treatment, 242 of 944 PwMS switched treatment again and 33 PwMS discontinued (selective) immunosuppressive treatment for the rest of the observation period (see ESM Table S2).

Information on switch reasons was available for $42.8 \%$ of the 944 patients who switched fingolimod treatment (Table 3). The most frequent reasons were disease activity (relapse/magnetic resonance imaging [MRI] activity; $44.1 \%$ ), adverse drug events (35.1\%; for example, lymphopenia), and patient request (7.7\%).

\section{Washout Periods and Relapse Activity}

Of the 944 PwMS who switched, 43 were excluded from subsequent analyses because of missing data on switch dates. Furthermore, 13 of the 19 different DMDs used after fingolimod were included in more detailed analyses due to statistically insufficient patient numbers in six post-fingolimod treatment groups $(N<10$, respectively: azathioprine, cyclophosphamide, intravenous immunoglobulin, mitoxantrone, other, ozanimod) (Table 4). Median washout periods after fingolimod discontinuation ranged from 1.0 to 3.0 months. The 901 treatmentswitching PwMS included in the analysis showed a slight increase in relapse activity during the washout period of fingolimod (ARR: pre-switch vs. washout 0.37 vs. 0.47 ), followed by a decrease after starting another treatment

Table 2 Patients using fingolimod in three treatment periods (2010-2013, 2014-2016, 2017-2019)

\begin{tabular}{|c|c|c|c|c|}
\hline Calendar periods & 2010-2013 & 2014-2016 & 2017-2019 & $p$ value \\
\hline$N(\%)$ & $886(34.9)$ & $968(38.2)$ & $682(26.9)$ & \\
\hline Women & $652(73.6)$ & $687(71.0)$ & $479(70.2)$ & $0.282^{\mathrm{d}}$ \\
\hline Age at fingolimod start (years) & $\begin{array}{l}39.1(31.7 \\
46.2)^{c}\end{array}$ & $38.5(30.9,46.6)^{\mathrm{c}}$ & $\begin{array}{l}38.7(30.5 \\
48.8)^{c}\end{array}$ & $0.587^{\mathrm{e}}$ \\
\hline Disease duration (years) ${ }^{\mathrm{a}}$ & $\begin{array}{r}8.5(4.4 \\
14.5)^{c}\end{array}$ & $7.0(3.1,13.5)^{\mathrm{c}}$ & $\begin{array}{r}7.1(3.0 \\
13.8)^{\mathrm{c}}\end{array}$ & $<0.001^{\mathrm{e}}$ \\
\hline \multirow[t]{2}{*}{ EDSS at fingolimod start ${ }^{\mathrm{b}}$} & $N=140$ & $N=465$ & $N=553$ & $<0.001^{\mathrm{e}}$ \\
\hline & $\begin{array}{l}2.5(1.5 \\
4.0)^{\mathrm{c}}\end{array}$ & $2.0(1.5,3.5)^{\mathrm{c}}$ & $\begin{array}{l}2.0(1.0 \\
3.5)^{\mathrm{c}}\end{array}$ & \\
\hline Therapy-naïve patients before fingolimod & $204(23.0)$ & $322(33.3)$ & $191(28.0)$ & $<0.001^{\mathrm{d}}$ \\
\hline $\begin{array}{l}\text { Number of DMDs before fingolimod initiation (mean } \pm \text { SD } \\
\text { [range]) }\end{array}$ & $\begin{array}{l}1.4 \pm 1.2 \\
{[0-7]}\end{array}$ & $\begin{array}{l}1.2 \pm 1.2 \\
{[0-6]}\end{array}$ & $\begin{array}{l}1.3 \pm 1.3 \\
{[0-7]}\end{array}$ & $0.003^{\mathrm{e}}$ \\
\hline $\begin{array}{l}\text { Proportion of patients taking fingolimod } \geq 1 \text { year after } \\
\text { therapy start }\end{array}$ & $794(89.6)$ & $838(86.6)$ & $549(80.5)$ & $<0.001^{\mathrm{d}}$ \\
\hline
\end{tabular}

$N$ (\%) Number of patients (proportion of patients)

${ }^{a}$ From MS onset until the start of fingolimod treatment

${ }^{\mathrm{b}}$ At latest visit within the year of fingolimod therapy initiation

${ }^{\mathrm{c}}$ Median (25\% quartile, $75 \%$ quartile)

${ }^{\mathrm{d}}$ Chi-square test

${ }^{\mathrm{e}}$ Kruskal-Wallis test 


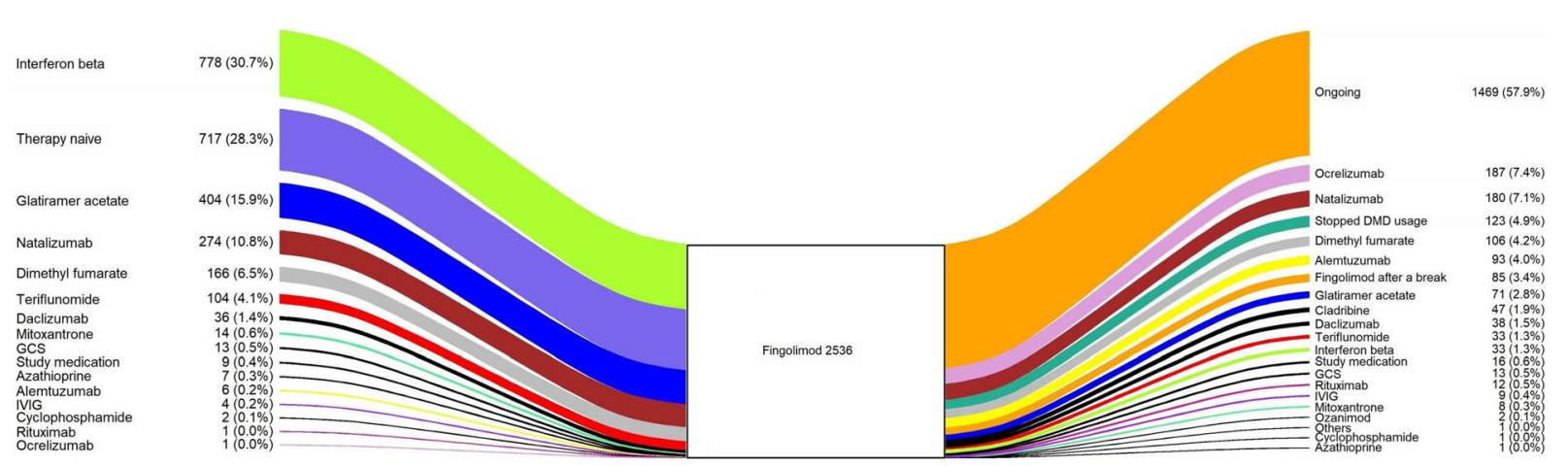

Fig. 2 Frequencies of disease-modifying drugs used prior to initiation of fingolimod treatment. The box in the middle represents the 2536 PwMS who started treatment with fingolimod. On the left side are shown the DMDs used prior to fingolimod initiation, with patient numbers and proportions. On the right side are listed the therapies after cessation of fingolimod treatment, with patient numbers and proportions. Patient groups were listed in descending order of frequency. The sizes of the colored lines correspond to the frequencies of the DMDs used. The largest group of patients was treated with interferon beta $(N=778)$ before starting fingolimod, followed by

Table 3 Reasons for treatment switches by 404 patients receiving fingolimod

\begin{tabular}{lc}
\hline Reason for switch & $\boldsymbol{N}(\%)$ \\
\hline Disease activity despite fingolimod treatment & $178(44.1)$ \\
Adverse drug event & $142(35.1)$ \\
Patient request & $31(7.7)$ \\
Childbearing preference & $18(4.5)$ \\
Therapy interruption & $10(2.5)$ \\
Poor therapy adherence & $6(1.5)$ \\
Pregnancy & $6(1.5)$ \\
Other & $13(3.2)$ \\
\hline
\end{tabular}

$N$ (\%) Number of patients (proportion of patients)

(ARR 0.19). The highest pre-switch ARR at the last visit when on fingolimod occurred in patients who later switched to alemtuzumab (ARR 0.63). During the washout period of fingolimod, the highest ARRs were observed in patients who later switched to study medication therapy-naïve patients $(N=717)$. Glatiramer acetate $(N=404)$, natalizumab $(N=274)$, and dimethyl fumarate $(N=166)$ were also frequently used as pre-fingolimod therapies. The majority of PwMS continued taking fingolimod until the end of the observation period $(N=1469)$. Patients who switched treatment mostly used ocrelizumab $(N=187)$, natalizumab $(N=180)$, or dimethyl fumarate $(N=106)$ after stopping fingolimod treatment. GCS Standard glucocorticosteroid pulse therapy, IVIG intravenous immunoglobulin

(ARR 0.69), natalizumab (ARR 0.66), and dimethyl fumarate (ARR 0.65). Relapse activity decreased after the start of the subsequent DMD in 11 of the 13 DMD patient groups analyzed compared to the treatment under fingolimod. PwMS who switched to interferon beta or the study medication showed an increase in ARR, respectively (pre-switch vs. post-switch: 0.36 vs. 0.44 for interferon beta, and 0.00 vs. 0.28 for the study medication). Additional information on time to first relapse during the washout period and after the start of the subsequent DMD is provided in ESM Table S3.

\section{Predictors of Switches and Relapses}

Cox regression models revealed that female and younger PwMS discontinued their fingolimod treatment significantly earlier than male (male: hazard ratio $[\mathrm{HR}] 0.78, p<0.001$; reference: female) and older patients (16-30 years of age: $\mathrm{HR}=1.27, p=0.003$; reference: $31-40$ years), respectively (Table 5). For example, 5 years after starting fingolimod treatment, $34.7 \%$ of male PwMS were still on treatment compared to 
Table 4 Clinical and therapeutic characteristics of fingolimod-switching patients

\begin{tabular}{|c|c|c|c|c|c|c|c|c|c|c|c|}
\hline \multirow{2}{*}{$\begin{array}{l}\text { Post- } \\
\text { fingolimod } \\
\text { treatment }^{a}\end{array}$} & \multirow[t]{2}{*}{$N(\%)$} & \multirow[t]{2}{*}{$F(\%)$} & \multirow[t]{2}{*}{$\begin{array}{l}\text { Age, years } \\
(\text { mean } \pm S D)^{c}\end{array}$} & \multirow{2}{*}{$\begin{array}{l}\text { WA of } \\
\text { fingolimod, } \\
\text { months }\end{array}$} & \multicolumn{4}{|c|}{ EDSS (median score) } & \multicolumn{3}{|c|}{$\begin{array}{l}\text { Annualized } \\
\text { relapse rate }\end{array}$} \\
\hline & & & & & $\overline{N^{c}}$ & Pre $^{c}$ & $N^{\mathrm{e}}$ & Post $^{\mathrm{e}}$ & Pre $^{c}$ & WA & Post $^{f}$ \\
\hline Total $^{\mathrm{b}}$ & $901(100.0)$ & 75.4 & $40.0 \pm 10.6$ & $2.0(1.0,5.0)$ & 458 & 3.0 & 429 & 3.0 & 0.37 & 0.47 & 0.19 \\
\hline Ocrelizumab & $178(19.8)$ & 71.9 & $41.8 \pm 10.3$ & $3.0(1.0,5.0)$ & 136 & 3.5 & 101 & 3.0 & 0.32 & 0.41 & 0.15 \\
\hline Natalizumab & $171(19.0)$ & 80.7 & $36.6 \pm 10.0$ & $2.0(1.0,4.0)$ & 79 & 2.5 & 74 & 2.5 & 0.39 & 0.66 & 0.17 \\
\hline $\begin{array}{l}\text { Dimethyl } \\
\text { fumarate }\end{array}$ & $99(11.0)$ & 69.7 & $40.4 \pm 9.5$ & $2.0(1.0,6.0)$ & 31 & 2.5 & 43 & 3.0 & 0.21 & 0.65 & 0.15 \\
\hline Alemtuzumab & $95(10.5)$ & 75.8 & $35.5 \pm 9.7$ & $3.0(2.0,4.5)$ & 33 & 3.0 & 45 & 3.0 & 0.63 & 0.53 & 0.15 \\
\hline $\begin{array}{l}\text { Fingolimod } \\
\text { after break }\end{array}$ & $85(9.4)$ & 72.9 & $40.3 \pm 11.0$ & n.a & 39 & 2.0 & 40 & 3.0 & 0.37 & n.a & 0.26 \\
\hline $\begin{array}{l}\text { Glatiramer } \\
\text { acetate }\end{array}$ & $67(7.4)$ & 79.1 & $42.9 \pm 11.5$ & $1.0(0.0,2.0)$ & 24 & 2.5 & 29 & 2.5 & 0.41 & 0.16 & 0.27 \\
\hline Cladribine & $46(5.1)$ & 78.3 & $39.9 \pm 10.4$ & $3.0(2.0,5.0)$ & 33 & 2.5 & 24 & 2.25 & 0.40 & 0.47 & 0.09 \\
\hline Daclizumab & $35(3.9)$ & 71.4 & $40.4 \pm 11.7$ & $2.0(1.0,2.5)$ & 19 & 3.0 & 26 & 3.0 & 0.52 & 0.00 & 0.34 \\
\hline Interferon beta & $33(3.7)$ & 78.8 & $39.4 \pm 11.7$ & $1.0(0.0,7.0)$ & 8 & 2.25 & 8 & 2.5 & 0.36 & 0.00 & 0.44 \\
\hline Teriflunomide & $33(3.7)$ & 87.9 & $45.3 \pm 9.2$ & $3.0(2.0,4.0)$ & 15 & 3.0 & 15 & 3.0 & 0.51 & 0.27 & 0.18 \\
\hline $\begin{array}{l}\text { Study } \\
\text { medication }\end{array}$ & $15(1.7)$ & 46.7 & $51.3 \pm 9.2$ & $1.0(0.0,6.0)$ & 15 & 4.0 & 5 & 5.0 & 0.00 & 0.69 & 0.28 \\
\hline GCS & $11(1.2)$ & 72.7 & $45.1 \pm 9.5$ & n.a & 8 & 4.75 & 4 & 6.25 & 0.11 & 0.35 & 0.00 \\
\hline Rituximab & $11(1.2)$ & 72.7 & $41.4 \pm 10.2$ & $2.0(2.0,3.0)$ & 9 & 3.0 & 15 & 3.0 & 0.44 & 0.50 & 0.22 \\
\hline
\end{tabular}

$F$ proportion of female patients, GCS regular glucocorticosteroid pulse therapy, $N(\%)$ number of patients (proportion of patients), $W A$ washout period

${ }^{a}$ Groups with statistically sufficient numbers of patients $(N \geq 10)$ are shown in detail

${ }^{b}$ We excluded 43 of the fingolimod-switching PwMS $(N=944)$ from this analysis due to missing data on switch dates

${ }^{\mathrm{c}}$ At last visit/during the last year under fingolimod treatment

${ }^{\mathrm{d}}$ Median (25\% quartile, $75 \%$ quartile)

e $6-12$ months after the initiation of the post-fingolimod treatment

${ }^{f}$ From the start of the post-fingolimod treatment until the end of the observation period

$30.6 \%$ of females. In patients aged $41-50$ years at the start of fingolimod treatment, $35.3 \%$ were still receiving fingolimod 5 years after treatment initiation, compared with $27.3 \%$ among those aged 16-30 years. No significant association was found for the time to discontinuation of fingolimod and EDSS score $(p \geq 0.419)$. Furthermore, sex, age (at last visit on fingolimod), degree of disability (at last visit on fingolimod), and length of washout period were not significantly associated with the time to the first relapse during the washout period of fingolimod or after starting the subsequent DMD $(p \geq 0.070)$. However, the time to the first relapse was twofold shorter in patients who switched than in patients who continued 
Table 5 Associations between time to discontinue fingolimod/first relapse and age, sex, disability level, and washout period

\begin{tabular}{|c|c|c|c|c|c|c|c|c|c|}
\hline \multirow[t]{2}{*}{ Characteristics } & \multicolumn{3}{|c|}{$\begin{array}{l}\text { Time to discontinuation of } \\
\text { fingolimod }\end{array}$} & \multicolumn{3}{|c|}{$\begin{array}{l}\text { Time to first relapse } \\
\text { during washout }\end{array}$} & \multicolumn{3}{|c|}{$\begin{array}{l}\text { Time to first relapse after } \\
\text { starting another DMD }\end{array}$} \\
\hline & $\overline{\text { HR }}$ & 95\% CI & $p$ & $\overline{\text { HR }}$ & 95\% CI & $p$ & $\overline{\text { HR }}$ & 95\% CI & $p$ \\
\hline \multicolumn{10}{|l|}{ Sex } \\
\hline Female & \multicolumn{3}{|c|}{ Reference } & \multicolumn{3}{|c|}{ Reference } & \multicolumn{3}{|c|}{ Reference } \\
\hline Male & 0.78 & $0.68-0.89$ & $<0.001^{*}$ & 0.82 & $0.47-1.43$ & 0.478 & 0.75 & $0.50-1.12$ & 0.165 \\
\hline Age (years) & \multicolumn{3}{|c|}{ At start of fingolimod treatment } & \multicolumn{3}{|c|}{$\begin{array}{l}\text { At last visit while on } \\
\text { fingolimod }\end{array}$} & \multicolumn{3}{|c|}{ At last visit while on fingolimoc } \\
\hline $16-30$ & 1.27 & $1.09-1.48$ & $0.003^{*}$ & 1.15 & $0.62-2.11$ & 0.659 & 1.19 & $0.77-1.82$ & 0.437 \\
\hline $31-40$ & \multicolumn{3}{|c|}{ Reference } & \multicolumn{3}{|c|}{ Reference } & \multicolumn{3}{|c|}{ Reference } \\
\hline $41-50$ & 0.90 & $0.77-1.05$ & 0.194 & 0.93 & $0.51-1.71$ & 0.825 & 1.16 & $0.75-1.77$ & 0.506 \\
\hline$\geq 51$ & 0.97 & $0.79-1.19$ & 0.789 & 0.64 & $0.30-1.39$ & 0.262 & 0.58 & $0.32-1.04$ & 0.070 \\
\hline EDSS & \multicolumn{3}{|c|}{$\begin{array}{l}\text { At latest visit within the year of } \\
\text { fingolimod treatment initiation }\end{array}$} & \multicolumn{3}{|c|}{$\begin{array}{l}\text { At last visit while on } \\
\text { fingolimod }\end{array}$} & \multicolumn{3}{|c|}{ At last visit while on fingolimoc } \\
\hline $0.0-3.5$ & 0.94 & $0.78-1.14$ & 0.547 & 1.28 & $0.62-2.62$ & 0.500 & 0.98 & $0.63-1.53$ & 0.935 \\
\hline $4.0-6.5$ & \multicolumn{3}{|c|}{ Reference } & \multicolumn{3}{|c|}{ Reference } & \multicolumn{3}{|c|}{ Reference } \\
\hline $7.0-8.0$ & 0.66 & $0.25-1.79$ & 0.419 & 0.95 & $0.12-7.47$ & 0.961 & 0.89 & $0.12-6.57$ & 0.911 \\
\hline \multicolumn{10}{|l|}{ Length of WA ${ }^{a}$} \\
\hline$\leq 6$ weeks & - & & & 1.70 & $0.59-4.92$ & 0.330 & 0.88 & $0.62-1.24$ & 0.459 \\
\hline$>6$ weeks & - & & & Refer & & & Refer & & \\
\hline
\end{tabular}

$C I$ confidence interval, $D M D$ disease-modifying drug, $H R$ hazard ratio

* Significant difference from Reference

${ }^{a}$ Complete elimination of fingolimod after 6 weeks at the earliest according to the summary of medicinal product characteristics (Gilenya ${ }^{\circledR}$ )

fingolimod treatment (HR 2.09, 95\% confidence interval 1.73-2.51, $p<0.001)$.

\section{DISCUSSION}

Analysis of patients taking fingolimod during the 2010-2013, 2014-2016, and 2017-2019 periods revealed several changes over time. First, the significant decrease in PwMS taking fingolimod continuously for $\geq 1$ year presumably reflects the increasing number of available treatment options, particularly in the early phase of treatment initiation. In addition, further insights were gained into the importance of pregnancy as a potentially protective period that could be reflected in clinical practice. Thus, physicians may have responded more liberally and discontinued fingolimod when patients raised questions about pregnancy planning. Second, there was an increase in the number of treatment-naïve PwMS starting fingolimod. Although the guidelines of the German Society of Neurology for the treatment of MS regarding the indication of fingolimod have remained unchanged over time [21, 22], except for patient 
age (in 2018 approval was expanded to 10- to 17 -year-olds [compared to only patients aged $\geq$ 18 years prior to 2018) [7]), an increasing level of pharmacovigilance data on the use of fingolimod has been accumulated from 2011 onwards. This growing body of evidence may have contributed to the fact that significantly more treatment-naïve patients were treated with fingolimod in the most recent study period than in the post-approval period.

Predictors of treatment switches in our study were young age and female sex. Generally, disease activity in the form of relapses is higher in patients of young age and decreases with age $[23,24]$. This trend is also indicated in the prospective, multicenter, longitudinal PANGEA study, which reported higher ARRs in younger fingolimod-treated patients with RRMS compared with older ones ( $\leq 20$ years: $2.0,>20$ to $\leq 30$ years: $1.7,>30$ years: 1.4.) [25]. Furthermore, DMDs of increased efficacy (e.g., fingolimod) have no additional effect on disability progression compared to more modest/moderate-efficacy DMDs (e.g., interferon beta) in PwMS aged > 40 years [26]. Data from the Danish MS Registry also showed that patients aged $<40$ years switched their initial treatment more frequently than older PwMS $(p \leq 0.008)$ [27]. The higher frequency of switching in females may be due to the known teratogenicity of fingolimod. When planning pregnancy, fingolimod should be discontinued $\geq 2$ months before conception. Reliable contraception is recommended both during discontinuation and during the 2-month washout phase [7]. One possibility to prevent a severe rebound during pregnancy after fingolimod cessation is to switch to rituximab before conception [28]. In the case of severe rebound after discontinuation of fingolimod and no or poor response to glucocorticosteroid (GCS) treatment, initiation of therapy with rituximab in pregnant women might be considered as an option, as described in a case study by Canibaño et al. [29]. Of course, the decision has to be preceded by a comprehensive risk-benefit assessment. Although therapy of a pregnant woman with a B cell-depleting antibody is controversial, the efficacy of rituximab cannot be dismissed, and this monoclonal antibody of the IgG1 isotype cannot cross the placenta in the first trimester [29]; only after the 16th week of pregnancy is the passage of rituximab across the placenta possible. However, the use of rituximab after discontinuation of fingolimod cannot be a general recommendation; rather, it should be carefully considered in each individual case.

An important risk factor that should be considered when stopping fingolimod treatment is the rebound effect, i.e., increased disease activity (relapses and/or T2- or gadoliniumenhancing lesions) after stopping fingolimod [30]. In a study by Hatcher et al., 46 PwMS were retrospectively analyzed for increased disease activity after discontinuing or switching fingolimod. Within 4-16 weeks after the treatment change, five patients (10.9\%) experienced severe relapses and an increase in new lesions [31]. Other studies with small case numbers reported similar phenomena in fingolimod-switching PwMS [32, 33]. Our analysis supports these findings: higher relapse rates occurred during the washout of fingolimod, particularly when PwMS switched to natalizumab, alemtuzumab, or dimethyl fumarate. In our study, as in other studies, no definitive predictors of increased relapse activity after cessation of fingolimod were identified [34]. Moreover, sex, age, degree of disability, and duration of washout period were not associated with the time to the first relapse during the washout period or after starting a subsequent DMD. However, it is advisable to monitor for increased relapse activity before switching, as this may be an indicator of a highly active disease course and could favor a rebound. In case of such an increased risk of disease reactivation after discontinuation of fingolimod, GCS might be used to bridge the washout period. In studies on the discontinuation of natalizumab, similar strategies were described in which high-dose GCS therapy was initiated at one day per month during the washout period $[35,36]$. Also, initiating treatment with high-efficacy DMDs, such as natalizumab or ocrelizumab, could significantly reduce the risk of rebound after the switch, as seen in our results. If the risk of rebound is assessed to be high, the duration of the period should be limited to the extent necessary. In addition, periodic MRI examinations 
can help to detect and treat signs of disease activity early [37] with, for example, GCS or plasma exchange [34].

Rebound risk after treatment discontinuation has to be balanced against adverse effects associated with a discontinued DMD, as the risk of severe drug--drug interactions is another important consideration when planning a therapy switch. Due to variability in the washout period of fingolimod and individual patient metabolism (for example, individual renal clearance and volume of distribution of drugs applied, which are dependent on age as well as on liver and kidney diseases), severe interactions of fingolimod (complete elimination after 6 weeks at the earliest) [7] with other DMDs, such as alemtuzumab, mitoxantrone, natalizumab, and teriflunomide, are possible. An increased risk of opportunistic infections is expected due to the additive immunosuppressive effects of fingolimod and those DMDs, with life-threatening PML (in human polyomavirus 2 [JC] virus-positive MS patients) or cryptococcal infections as hazard scenarios [38, 39]. A benefit-risk assessment should be performed before switching the fingolimod treatment. Our analysis revealed that the time to the first relapse after fingolimod cessation was not significantly associated with the length of the washout period. Therefore, in case of switching the treatment, an individualized washout period should be preferred over a generalized one, taking into account the patient's current disease activity, pregnancy planning, and the possibility of prolonged lymphopenia [40]. Generally, the mechanism of action as well as efficacy of the DMD to be switched should also be included in the planning of an adequate washout period. According to our data, ARR was higher only after the start of the post-fingolimod treatments with interferon beta, daclizumab, and glatiramer acetate compared with the washout period. Especially for these DMDs, regular followup appointments with the treating neurologist should be a requirement after fingolimod cessation, even after initiation of the post-fingolimod therapy. However, it should also be considered that the patient numbers in the analysis of these three DMDs was $<70$, respectively (see Table 4). Additional analyses with larger patient groups are needed to validate these results before they can be used as the basis of specific recommendations for action. Generally, a washout period of at least six weeks (eight weeks when planning a pregnancy) is recommended for fingolimod [7], during which the patient should be examined regularly for signs of rebound.

In terms of treatments prior to the initiation of fingolimod, common treatment strategies include interferon beta (escalation), glatiramer acetate (escalation), natalizumab (de-escalation), or dimethyl fumarate (escalation). In the case of natalizumab, the significantly higher PML risk in PwMS with a positive JC virus antibody index compared to fingolimod may favor the decision to switch to fingolimod. Possible formation of neutralizing antibodies can also lead to reduced (selective) immunosuppressive efficacy of natalizumab and should result in a therapy switch in the first months of natalizumab therapy [41]. Furthermore, a switch from natalizumab to fingolimod should be conducted within 12 weeks after cessation of natalizumab due to the lower risk of disease reactivation, as suggested by previous studies $[35,42]$. Another advantage of fingolimod compared to the three most common pre-therapies is the administration route, as fingolimod is administered orally while the other three DMDs are administered by injection or infusion.

Natalizumab and ocrelizumab are generally highly effective in decreasing relapse activity as well as in slowing disability progression [43]. Therefore, the choice of these monoclonal antibodies as the most common post-fingolimod therapies in our study is plausible in cases with high disease activity. These findings are further supported by the reasons for switching fingolimod that were reported for a subgroup of 404 PwMS. The most common reason was disease activity (relapses or disease activity revealed by MRI) in 178 PwMS (44.1\%) indicating insufficient efficacy of treatment with fingolimod. A similar finding was recorded in a longitudinal study examining patients with relapsing MS treated with fingolimod: over a 36-month period, relapses were detected in $41.8 \%$ of the 1571 patients [44]. In a study on 
cessation of fingolimod treatment, $55.8 \%$ of the 230 MS patients included in the analysis reported insufficient efficacy of fingolimod as a reason for discontinuation [45]. In a cross-sectional study that examined the reasons for switching in 595 patients with RRMS taking mildly or moderately effective DMDs, the most common reason was failure of the current therapy (53.9\%) [46]. If disease activity is present despite (selective) immunosuppressive therapy, a rapid switch to more effective DMDs, such as monoclonal antibodies (e.g., natalizumab or ocrelizumab), represents an appropriate option. Nevertheless, switching to monoclonal antibodies should be weighed on an individual basis, as the selectivity and high efficacy are also offset by the risk of severe adverse drug events [47]. For example, PML in patients on natalizumab and eventual respiratory tract infections in those on ocrelizumab should be considered when switching to a new treatment [47], as adverse drug events were the second most frequent reason for therapy changes $(35.1 \%)$ in our study. A further point to be considered is the proportion of patients who did not started a subsequent DMD after the cessation of fingolimod. In our study, this applied to $4.9 \%$ of all patients. In a U.S. study that assessed 535 patients on fingolimod for treatment switches, the proportion of such patients was $7.5 \%$ [48], similar to our study. In a sociodemographically and clinically comparable population of 230 Italian patients with RRMS, the proportion of patients without reinitiation of therapy after fingolimod discontinuation was higher at $12.6 \%$ [45]. In general, the proportion of patients without new therapy initiation is relatively low. Differences between studies may result from the varying study designs (multicenter vs. single center), the different size of the study populations, and the international diversity of the health care setting.

There are some strengths and limitations to the present study. The reasons for switching from fingolimod therapy were only available for a subset of 404 PwMS, which limits the possibility of interpretation. However, data from this subset represent $>40 \%$ of all PwMS who switched from fingolimod to other DMDs in our study. Furthermore, only limited data were available on drug safety related to treatment switches, such as infection rates or lymphocyte numbers. Data on pharmacovigilance comprising adverse events, pregnancy, body mass, and medical history as well as data on the reasons for switching are only reported by the GMSR since 2019 [13]. Consequently, data on those reasons were limited during the observation period. From 2019, this new dataset steadily increased to date, and in the future this dataset will expand and allow for more extensive investigations of switching behavior and reasons. Moreover, the proportion of fingolimod patients is expected to decrease in the coming years due to the approval of the more selective binding S1PR modulators siponimod, ozanimod, and ponesimod. Our study is distinguished from previously published studies on therapy changes in fingolimod patients $[45,49]$ by including $>2500$ MS patients who were studied for sociodemographic and clinical changes over the largest and most recent observation period, identifying both prior and subsequent fingolimod therapies in a representative cohort of MS patients, breaking down the reasons for switching, and additionally identifying patient characteristics associated with switches and relapses.

\section{CONCLUSIONS}

In conclusion, our analysis revealed that most PwMS remained on fingolimod therapy. Young age ( $\leq 30$ years) and female sex were identified as predictors of early treatment switches in fingolimod-treated patients. Our study also underlines that therapy switches from fingolimod to another DMD occur after a shorter treatment duration today than in the years directly following fingolimod approval. On the one hand, it should be considered that more available treatment options lead to more frequent medication switches, possibly making the treatment of PwMS more complex and increasing the risk of polypharmacy [50]. In addition, cumulative risks associated with a sequential use of drugs that suppress the immune system in different ways and to different extents are an issue that is largely unexplored. On the other 
hand, a growing spectrum of treatments offers the possibility to respond better and faster to the healthcare needs (medical and personal) of the individual patient being treated. Our study also supports findings that there is an increased risk of rebound effects after switching from fingolimod and that the time to the first relapse was significantly shorter in the patients who switched than in those who remained on fingolimod, which adversely impacts disease progression. Although no clear predictors of relapse activity after the discontinuation of fingolimod could be identified in our study, planning for an adequate washout period of fingolimod before starting new treatments is essential due to the additive immunosuppressive effects of fingolimod remaining in the body and newly initiated DMDs. Finally, we observed an increasing willingness to discontinue fingolimod treatment in favor of starting another DMD over time among the analyzed patients and their physicians. For this reason, it is important to weigh the benefits and risks of any change in therapy in order to offer patients the best possible therapeutic options. Future studies should focus on drug safety outcomes of fingolimod patients in a longitudinal setting regarding adverse events (such as infections, macular degeneration, or leucopenia), disability, pregnancy, and no evidence of disease activity.

\section{ACKNOWLEDGEMENTS}

We would like to thank all patients that have given their informed consent. Furthermore, this study would not have been possible without the efforts of the centers participating in the registries. The centers are listed in the ESM.

Funding. The German MS Registry of the German MS Society was initiated and funded by the German MS Foundation and the German MS Society in 2001. It is operated by a not-forprofit company, the MSFP. MSFP receives funding from a broad range of public and private sponsors, including the German MS Society, the German MS Foundation, the Innovation Fund of the German Federal Joint
Committee, and the German Retirement Insurance. In 2021, Biogen, Celgene (BMS), Merck, Novartis, Roche and Sanofi participated in the multi-stakeholder funding approach to support the registry's operation and to allow the collection and reporting of (pharmacovigilance) data required as part of the EMA-minimal data set. Industry funding does not result in restrictions to publishing data, nor do the funders have access to the raw data or have any influence over the scientific conduct of the registry. The Journal's Rapid Service Fee was funded by the authors.

Authorship. All authors meet the International Committee of Medical Journal Editors (ICMJE) criteria for authorship for this article. All authors had full access to the study data and take responsibility for the integrity of the data and the accuracy of the data analysis, and have given their approval for this version to be published.

Author Contributions. AS designed and conceptualized study, revised the manuscript for intellectual content, and played a major role in the acquisition of data. CK revised the manuscript for intellectual content. CW revised the manuscript for intellectual content. DE designed and conceptualized study, analyzed the data, and revised the manuscript for intellectual content. DK revised the manuscript for intellectual content. FF analyzed the data. FP revised the manuscript for intellectual content. $\mathrm{JH}$ revised the manuscript for intellectual content. $\mathrm{KH}$ revised the manuscript for intellectual content. NF wrote the original draft and interpreted the data. PF revised the manuscript for intellectual content. PSR designed and conceptualized study and revised the manuscript for intellectual content. TP revised the manuscript for intellectual content. UKZ designed and conceptualized study and revised the manuscript for intellectual content.

Disclosures. David Ellenberger, Dagmar Krefting, Firas Fneish, Judith Haas, Kerstin Hellwig, and Tina Parciak declare no competing interests relevant to the content of the submitted manuscript. Niklas Frahm received travel 
funds for research meetings from Novartis; none resulted in a conflict of interest. Peter Flachenecker has received speaker's fees and honoraria for advisory boards from Almirall, Bayer, Biogen Idec, Celgene, Genzyme, Novartis, Merck-Serono, Roche and Teva and has participated in pharmaceutical company sponsored trials by Roche; none resulted in a conflict of interest. Friedemann Paul has received speaking fees, travel support, honoraria from advisory boards, and/or financial support for research activities from Bayer, Novartis, Biogen, Teva, Sanofi-Aventis/Genzyme, Merck Serono, Alexion, Chugai, MedImmune, Shire, German Research Council, Werth Stiftung of the City of Cologne, German Ministry of Education and Research, EU FP7 Framework Program, Arthur Arnstein Foundation Berlin, Guthy Jackson Charitable Foundation, and National Multiple Sclerosis of the USA. He also serves as academic editor for PLOS ONE and associate editor for Neurology, Neuroimmunology and Neuroinflammation. None of these activities has resulted in a conflict of interest. Clemens Warnke has received institutional support from Novartis, Biogen, Alexion, Janssen, and Roche; none resulted in a conflict of interest. Christoph Kleinschnitz has received speaker's fees, honoraria for attending advisory boards, and financial support for conducting research projects from Merck Serono GmbH, Germany and Merck KGaA, Germany; none resulted in a conflict of interest. Paulus S. Rommer has received speaking fees, honoraria from advisory boards, and/or financial support for research activities from AbbVie, Amicus, Biogen, Daiichi-Sankyo, Merck Serono, Novartis, Roche, Sandoz, Sanofi Genzyme, and Teva; none resulted in a conflict of interest. Alexander Stahmann has no personal pecuniary interests to disclose, other than being the lead of the German MS Registry, which receives funding from a range of public and corporate sponsors, recently including The German Innovation Fund (G-BA), The German MS Trust, The German MS Society, The German Retirement Insurance, Biogen, Celgene (BMS), Merck, Novartis, Roche, and Sanofi. Uwe K. Zettl has received speaking fees, travel support and /or financial support for research activities from Alexion, Almirall, Bayer, Biogen, Bristol-
Myers-Squibb, Janssen, Merck Serono, Novartis, Octapharm, Roche, Sanofi Genzyme, Teva as well as EU, BMBF, BMWi and DFG; none resulted in a conflict of interest.

Compliance with Ethics Guidelines. The GMSR was registered with the German Register of Clinical Studies (DRKS, Deutsches Register Klinischer Studien, DRKS; No. DRKS00011257). Institutional review board at the University of Würzburg obtained initial ethical approval (permit number 142/12).

Data Availability. Data of the GMSR is administered by MS Research and Project Development gGmbH. Anonymized data will be made available on request for any qualified investigator under the terms of the registries' usage and access guidelines and subject to the informed consent of the patients.

Open Access. This article is licensed under a Creative Commons Attribution-NonCommercial 4.0 International License, which permits any non-commercial use, sharing, adaptation, distribution and reproduction in any medium or format, as long as you give appropriate credit to the original author(s) and the source, provide a link to the Creative Commons licence, and indicate if changes were made. The images or other third party material in this article are included in the article's Creative Commons licence, unless indicated otherwise in a credit line to the material. If material is not included in the article's Creative Commons licence and your intended use is not permitted by statutory regulation or exceeds the permitted use, you will need to obtain permission directly from the copyright holder. To view a copy of this licence, visit http://creativecommons.org/licenses/by$\mathrm{nc} / 4.0 /$.

\section{REFERENCES}

1. Dobson R, Giovannoni G. Multiple sclerosis-a review. Eur J Neurol. 2019;26:27-40.

2. Aktas O, Renner A, Huss A, et al. Serum neurofilament light chain: no clear relation to cognition and 
neuropsychiatric symptoms in stable MS. Neurol Neuroimmunol Neuroinflamm. 2020;7:e885.

3. Rommer PS, Eichstädt K, Ellenberger D, et al. Symptomatology and symptomatic treatment in multiple sclerosis: results from a nationwide MS registry. Mult Scler. 2019;25:1641-52.

4. Ellenberger D, Stahmann A, Flachenecker P, et al. [Differences in the time to first disease-modifying treatment for patients with relapsing-remitting multiple sclerosis in Germany]. Congress of the German Society of Neurology; 2019.https://www. dgnvirtualmeeting.org/\#!resources/unterschiede-in -der-zeit-bis-zur-ersten-krankheitsmodifizierendent herapie-bei-ms-erkrankten-mit-schubformigem-ver lauf-in-deutschland

5. Thompson AJ, Baranzini SE, Geurts J, Hemmer B, Ciccarelli O. Multiple sclerosis. Lancet. 2018;391: 1622-36.

6. Thomas K, Proschmann U, Ziemssen T. Fingolimod hydrochloride for the treatment of relapsing remitting multiple sclerosis. Expert Opin Pharmacother. 2017;18:1649-60.

7. European Medicines Agency. Gilenya. Summary of product characteristics. 2021. https://www.ema. europa.eu/en/medicines/human/EPAR/gilenya. Accessed 6 Jan 2022

8. U.S. Food and Drug Administration. Novartis. Gilenya. Summary of product characteristics. 2019. https://www.accessdata.fda.gov/drugsatfda_docs/ label/2019/022527s26lbl.pdf. Accessed 6 Jan 2022

9. Cohen JA, Barkhof F, Comi G, et al. Oral fingolimod or intramuscular interferon for relapsing multiple sclerosis. N Engl J Med. 2010;362:402-15.

10. You Y, Barnett MH, Yiannikas C, et al. Interferon- $\beta$ is less effective than other drugs in controlling the rate of retinal ganglion cell loss in MS. Neurol Neuroimmunol Neuroinflamm. 2021;8:e971.

11. Rommer PS, Zettl UK. Managing the side effects of multiple sclerosis therapy: pharmacotherapy options for patients. Expert Opin Pharmacother. 2018;19:483-98.

12. Jalusic KO, Ellenberger D, Rommer P, Stahmann A, Zettl U, Berger K. Effect of applying inclusion and exclusion criteria of phase III clinical trials to multiple sclerosis patients in routine clinical care. Mult Scler. 2021;27:1852-63.

13. Ohle LM, Ellenberger D, Flachenecker P, et al. Chances and challenges of a long-term data repository in multiple sclerosis: 20th birthday of the German MS registry. Sci Rep. 2021;11:13340.
14. Haas J, Linker RA, Hartung HP, Meergans M, Ortler S. Tracik F [Fingolimod compassionate use program: case study on the concept of a therapy option for multiple sclerosis prior to marketing approval]. Nervenarzt. 2012;83:1575-81.

15. European Medicines Agency. Lemtrada. Summary of product characteristics. 2021. https://www.ema. europa.eu/en/medicines/human/EPAR/lemtrada. Accessed 6 Jan 2022

16. Samjoo IA, Worthington E, Drudge C, et al. Efficacy classification of modern therapies in multiple sclerosis. J Comp Eff Res. 2021;10:495-507.

17. European Medicines Agency. Tecfidera. Summary of product characteristics. 2021. https://www.ema. europa.eu/en/medicines/human/EPAR/tecfidera. Accessed 6 Jan 2022

18. European Medicines Agency. Zinbryta. Summary of product characteristics. 2018. https://www.ema. europa.eu/en/medicines/human/EPAR/zinbryta. Accessed 6 Jan 2022

19. European Medicines Agency. Mavenclad. Summary of product characteristics. 2021. https://www.ema. europa.eu/en/medicines/human/EPAR/mavenclad. Accessed 6 Jan 2022

20. European Medicines Agency. Ocrevus. Summary of product characteristics. 2021. https://www.ema. europa.eu/en/medicines/human/EPAR/ocrevus. Accessed 6 Jan 2022

21. Hemmer B. Diagnose und Therapie der Multiplen Sklerose, Neuromyelitis-opticaSpektrum-Erkrankun gen und MOG-IgG-assoziierten Erkrankungen. 2021. https://dgn.org/wp-content/uploads/2021/04 /030050_LL_Multiple_Sklerose_2021.pdf. Accessed 6 Jan 2022

22. Gold R. Diagnose und Therapie der Multiplen Sklerose, Neuromyelitis-opticaSpektrum-Erkrankun gen und MOG-IgG-assoziierten Erkrankungen. 2014. https://dgn.org/wp-content/uploads/2013/01 /030-0501_S2e_Multiple_Sklerose_Diagnostik_Thera pie_Archiv-min.pdf. Accessed 6 Jan 2022

23. Gorman MP, Healy BC, Polgar-Turcsanyi M, Chitnis $\mathrm{T}$. Increased relapse rate in pediatric-onset compared with adult-onset multiple sclerosis. Arch Neurol. 2009;66:54-9.

24. Fay AJ, Mowry EM, Strober J, Waubant E. Relapse severity and recovery in early pediatric multiple sclerosis. Mult Scler. 2012;18:1008-12.

25. Ziemssen T, Albrecht H, Haas J, et al. Descriptive analysis of real-world data on fingolimod long-term treatment of young adult RRMS patients. Front Neurol. 2021;12:637107. 
26. Weideman AM, Tapia-Maltos MA, Johnson K, Greenwood M, Bielekova B. Meta-analysis of the age-dependent efficacy of multiple sclerosis treatments. Front Neurol. 2017;8:577.

27. Sorensen PS, Kopp TI, Joensen H, Olsson A, Sellebjerg F, Magyari M. Age and sex as determinants of treatment decisions in patients with relapsingremitting MS. Mult Scler Relat Disord. 2021;50: 102813.

28. Smith JB, Hellwig K, Fink K, Lyell DJ, Piehl F, Langer-Gould A. Rituximab, MS, and pregnancy. Neurol Neuroimmunol Neuroinflamm. 2020;7:e734.

29. Canibaño B, Ali M, Mesraoua B, et al. Severe rebound disease activity after fingolimod withdrawal in a pregnant woman with multiple sclerosis managed with rituximab: a case study. Case Rep Womens Health. 2020;25:e00162.

30. Freedman MS, Selchen D, Prat A, Giacomini PS. Managing multiple sclerosis: treatment initiation, modification, and sequencing. Can J Neurol Sci. 2018;45:489-503.

31. Hatcher SE, Waubant E, Nourbakhsh B, CrabtreeHartman E, Graves JS. Rebound syndrome in patients with multiple sclerosis after cessation of fingolimod treatment. JAMA Neurol. 2016;73: 790-4.

32. Berger B, Baumgartner A, Rauer S, et al. Severe disease reactivation in four patients with relapsingremitting multiple sclerosis after fingolimod cessation. J Neuroimmunol. 2015;282:118-22.

33. Willis $\mathrm{M}$, Pearson $\mathrm{O}$, Illes $\mathrm{Z}$, et al. An observational study of alemtuzumab following fingolimod for multiple sclerosis. Neurol Neuroimmunol Neuroinflamm. 2017;4:e320.

34. Barry B, Erwin AA, Stevens J, Tornatore C. Fingolimod rebound: a review of the clinical experience and management considerations. Neurol Ther. 2019;8:241-50.

35. Havla J, Kleiter I, Kümpfel T. Bridging, switching or drug holidays - how to treat a patient who stops natalizumab? Ther Clin Risk Manag. 2013;9:361-9.

36. Fuentes-Rumí L, Hernández-Clares R, CarreónGuarnizo E, et al. Prevention of rebound effect after natalizumab withdrawal in multiple sclerosis. Study of two high-dose methylprednisolone schedules. Mult Scler Relat Disord. 2020;44:102311.

37. Sato K, Niino M, Kawashima A, Yamada M, Miyazaki Y, Fukazawa T. Disease exacerbation after the cessation of fingolimod treatment in japanese patients with multiple sclerosis. Intern Med. 2018;57:2647-55.
38. Ma SB, Griffin D, Boyd SC, Chang CC, Wong J, Guy SD. Cryptococcus neoformans var grubii meningoencephalitis in a patient on fingolimod for relapsing-remitting multiple sclerosis: case report and review of published cases. Mult Scler Relat Disord. 2020;39:101923.

39. Zhai S, Brew BJ. Progressive multifocal leukoencephalopathy. Handb Clin Neurol. 2018;152: 123-37.

40. Nagy S, Kuhle J, Derfuss T. Lymphocyte recovery after fingolimod discontinuation in patients with MS. Neurol Neuroimmunol Neuroinflamm. 2020;7: e874.

41. European Medicines Agency. Tysabri. Summary of product characteristics. 2021. https://www.ema. europa.eu/en/medicines/human/EPAR/tysabri. Accessed 6 Jan 2022

42. Sellner J, Rommer PS. A review of the evidence for a natalizumab exit strategy for patients with multiple sclerosis. Autoimmun Rev. 2019;18:255-61.

43. Rae-Grant A, Day GS, Marrie RA, et al. Practice guideline recommendations summary: diseasemodifying therapies for adults with multiple sclerosis: report of the Guideline development, dissemination, and implementation subcommittee of the American Academy of Neurology. Neurology. 2018;90:777-88.

44. Ziemssen T, Lang M, Tackenberg B, et al. Real-world persistence and benefit-risk profile of fingolimod over 36 months in Germany. Neurol Neuroimmunol Neuroinflamm. 2019;6:e548.

45. Landi D, Signori A, Cellerino M, Fenu G, Nicoletti $\mathrm{CG}$, Ponzano $\mathrm{M}$, et al. What happens after fingolimod discontinuation? A multicentre real-life experience. J Neurol. 2021. https://doi.org/10.1007/ s00415-021-10658-8.

46. Mäurer M, Tiel-Wilck K, Oehm E, et al. Reasons to switch: a noninterventional study evaluating immunotherapy switches in a large German multicentre cohort of patients with relapsing-remitting multiple sclerosis. Ther Adv Neurol Disord. 2019;12:1756286419892077.

47. Jalkh G, Abi Nahed R, Macaron G, Rensel M. Safety of newer disease modifying therapies in multiple sclerosis. Vaccines. 2020;9:12.

48. Vollmer B, Ontaneda D, Harris H, et al. Comparative discontinuation, effectiveness, and switching practices of dimethyl fumarate and fingolimod at 36-month follow-up. J Neurol Sci. 2019;407: 116498. 
49. Ziemssen T, Schulze-Topphoff U. The change of fingolimod patient profiles over time: a descriptive analysis of two non-interventional studies PANGAEA and PANGAEA 2.0. J Pers Med. 2021;11:561.
50. Frahm N, Hecker M, Zettl UK. Polypharmacy among patients with multiple sclerosis: a qualitative systematic review. Expert Opin Drug Saf. 2020;19:139-45. 